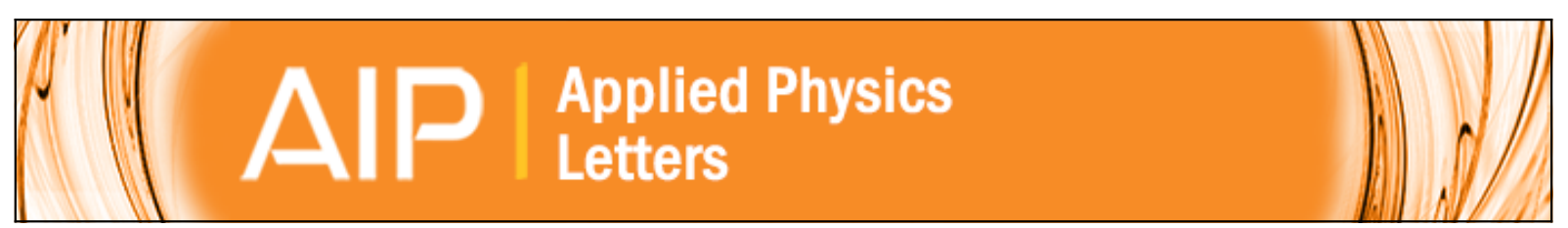

Determination of transient atomic structure of laser-excited materials from timeresolved diffraction data

Yvelin Giret, Nobuyasu Naruse, Szymon L. Daraszewicz, Yoshie Murooka, Jinfeng Yang, Dorothy M. Duffy, Alexander L. Shluger, and Katsumi Tanimura

Citation: Applied Physics Letters 103, 253107 (2013); doi: 10.1063/1.4847695

View online: http://dx.doi.org/10.1063/1.4847695

View Table of Contents: http://scitation.aip.org/content/aip/journal/apl/103/25?ver=pdfcov

Published by the AIP Publishing

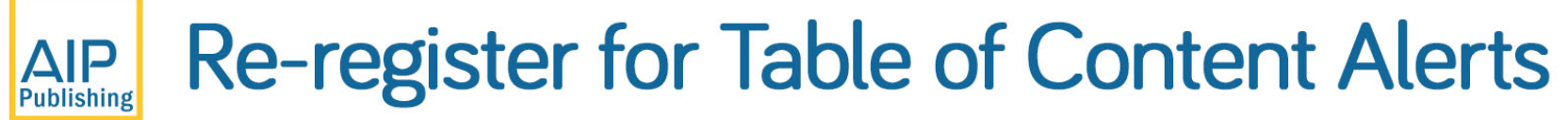

Create a profile. 


\title{
Determination of transient atomic structure of laser-excited materials from time-resolved diffraction data
}

\author{
Yvelin Giret, ${ }^{1,2}$ Nobuyasu Naruse, ${ }^{1}$ Szymon L. Daraszewicz, ${ }^{2}$ Yoshie Murooka, ${ }^{1}$ \\ Jinfeng Yang, ${ }^{1}$ Dorothy M. Duffy, ${ }^{2}$ Alexander L. Shluger, ${ }^{2}$ and Katsumi Tanimura ${ }^{1}$ \\ ${ }^{1}$ The Institute of Scientific and Industrial Research (ISIR), Osaka University, Mihogaoka 8-1, Ibaraki, \\ Osaka 567-0047, Japan \\ ${ }^{2}$ Department of Physics and Astronomy, University College London, Gower Street, WC1E 6BT London, \\ United Kingdom
}

(Received 23 August 2013; accepted 25 November 2013; published online 17 December 2013)

\begin{abstract}
The time evolution of the Bragg peaks of photo-excited gold nanofilms is measured using transmission ultrafast electron diffraction (UED) with $3.0 \mathrm{MeV}$ electron pulses and the corresponding structure evolution is calculated using two-temperature molecular dynamics (2TMD). The good agreement obtained between the measured and calculated Bragg peaks, over the full experimental timescale, enables the lattice temperature effects and the structural changes to be disentangled for the first time. The agreement demonstrates that 2T-MD is a reliable method for solving the inverse problem of structure determination of laser irradiated metals in UED measurements. (C) 2013 AIP Publishing LLC. [http://dx.doi.org/10.1063/1.4847695]
\end{abstract}

Recent advances in ultrafast diffraction techniques ${ }^{1,2}$ have enabled the study of the time evolution of crystal structures and have opened up the exciting prospect of unravelling the mechanisms of solid/solid and solid/liquid phase transitions. However, progress towards quantitative structure determination from ultrafast diffraction has been hindered by the absence of a reliable scheme to invert the excited-state diffraction data to provide the time evolution of the threedimensional atomic structure. ${ }^{3}$ Determining the structural changes during solid/liquid transformations is particularly challenging because of the rapid evolution and the disorder of the ensuing structure. The Progressive Reverse Monte Carlo Method has been used to visualise the dynamics of the surface premelting of excited Au nanocrystals ${ }^{3}$ whereas the ultrafast solid-liquid phase transition dynamics in $\mathrm{Al}$ has been expressed in terms of an average pair correlation function. ${ }^{4}$ The intensities of Bragg peaks measured in ultrafast electron diffraction (UED) experiments reflect both a lattice temperature effect, in terms of the Debye-Waller factor (DWF), and details of the structural changes in the solid. ${ }^{5}$ However, these two contributions have not been disentangled in previous experiments because the theoretical modelling $^{6,7}$ has been based on a continuum two-temperature model, which does not reveal the atomistic dynamics explicitly.

In this Letter, we use the hybrid method that combines the two-temperature model with classical molecular dynamics $(2 \mathrm{~T}-\mathrm{MD})^{8,9}$ to unravel the real time atomistic dynamics and melting mechanisms of excited gold nanofilms. This method has already been used to model the laser-induced melting of gold nanofilms ${ }^{8,10}$ and nanorods; ${ }^{11}$ however, direct comparison between theory and experiment has not previously been achieved. By comparing the theoretical predictions with the experimental UED measurements, we demonstrate that the application of 2T-MD to the determination of the structural evolution of photo-excited metal nanofilms is equivalent to solving the inverse problem for the timeevolution of structure from the transmission UED data. The results also serve as a strong foundation for detailed tests of the 2T-MD model.

Interpretation of UED data is more straightforward when metallic films are monocrystalline and thin enough to avoid dynamic scattering effects. In this work, we used single crystal gold thin-films with thickness of $10 \pm 2 \mathrm{~nm}$ which were placed on a gold mesh and inserted into the diffraction chamber at $293 \mathrm{~K}$. The films were excited at the incident angle of $14^{\circ}$ to the surface normal with $3.1 \mathrm{eV}$ photon pulses having a 90 fs pulse width at half maximum. Transmission electron diffraction was measured with relativistic $3.0 \mathrm{MeV}$ electron pulses with a 95 fs pulse width at half maximum. The diameter of the probed region $(0.2 \mathrm{~mm})$ is significantly smaller than that of the excited region $(0.8 \mathrm{~mm})$ and the electron beam is probing only the central homogenously excited part of the sample. The temporal resolution of pump-probe measurement was $180 \mathrm{fs}$, including timing jitter between laser and electron pulses.
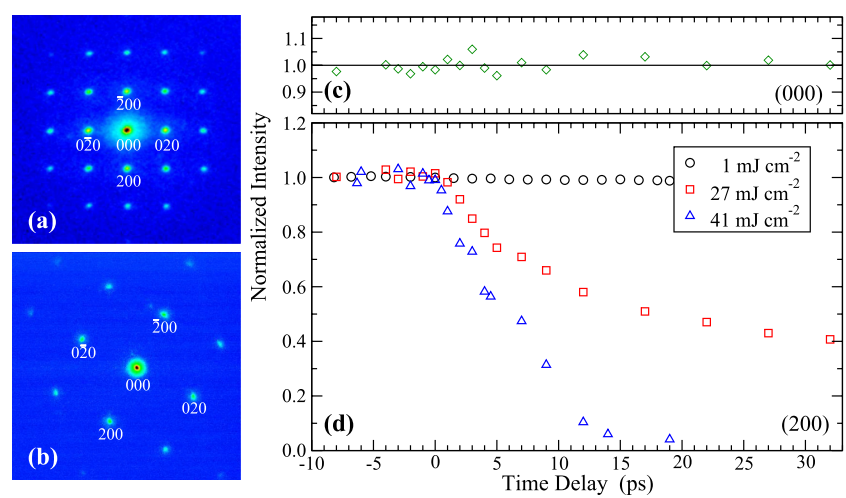

FIG. 1. Single shot diffraction images (UED) measured by two different lens modes: a wide-momentum mode (a) and a high-resolution mode (b). The scattering vectors of (200) and (220) peaks are 0.490 and $0.693 \AA^{-1}$, respectively. (c) Typical time-evolution of the (000)-order peak obtained by single-shot measurement at $F=27 \mathrm{~mJ} \mathrm{~cm}^{-2}$. (d) Evolution of the (200) Bragg peak intensity of a single crystal gold film under three different incident fluences, $F=1,27$ and $41 \mathrm{~mJ} \mathrm{~cm}^{-2}$. The intensity averaged over four equivalent (200) Bragg-peak spots is plotted. 

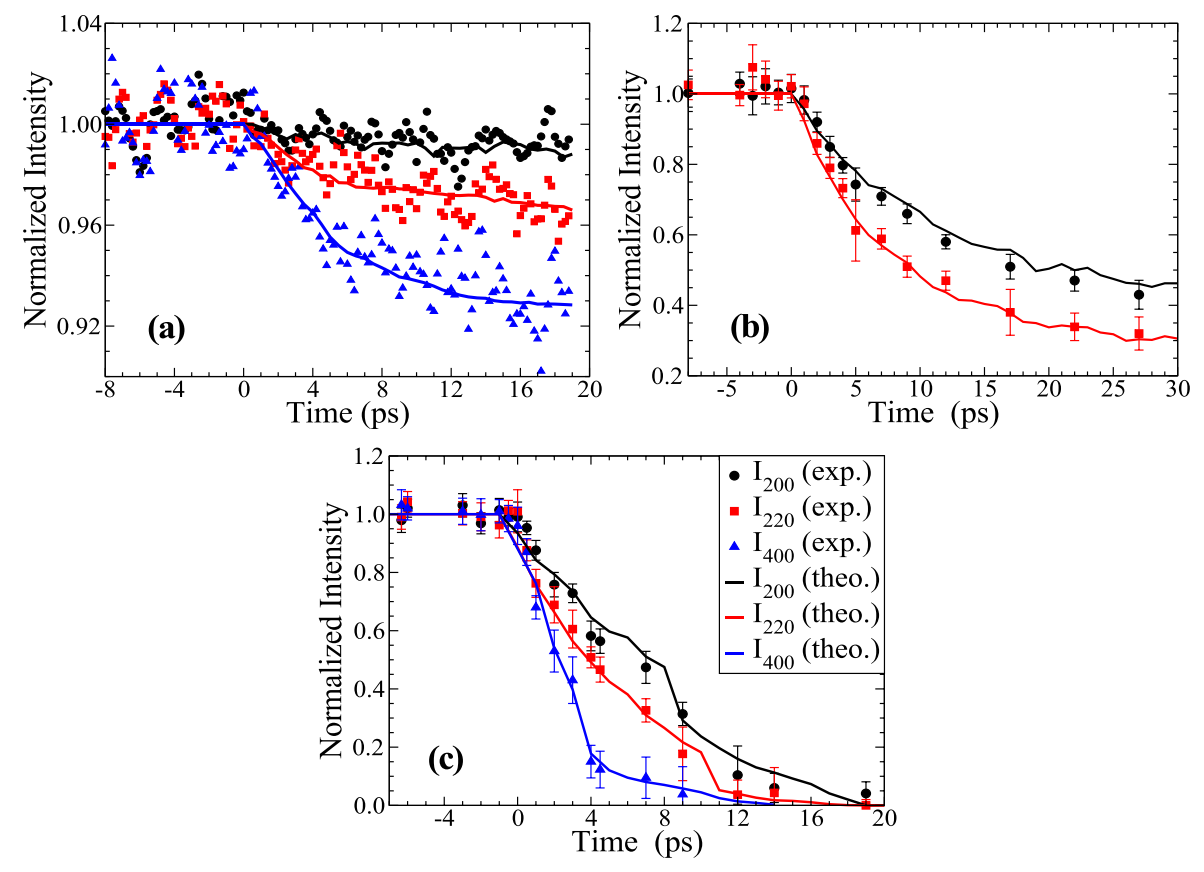

Our structure prediction strongly depends on the quality of experimental data. The relativistic electron energy of the probe beam gives two crucial advantages over conventional UED systems. ${ }^{6}$ First, space-charge effects are minimized and this allows us to perform high-quality single shot measurements, while maintaining the pulse width less than 100 fs. As illustrated in Fig. 1(a), higher-order spots up to a maximum scattering vector exceeding $1.56 \AA^{-1}$ can be captured clearly in a single shot. Second, our diffractometer provides structural information almost free from any multiple diffraction and possible inelastic effects. For 3.0 MeV electrons, the extinction distance for (200)-order in $\mathrm{Au}$ is $186 \mathrm{~nm}$, much larger than the sample thickness $(10 \mathrm{~nm})$; hence multiple diffraction effects can be neglected. ${ }^{12,13}$ In fact, the (000)-order peak intensity remains constant (Fig. 1(c)) in our measurements, hence the kinematic theory that assumes single scattering events, can be applied. Some of the previous UED measurements exhibit transient (000)-order attenuation, characteristic of multiple scattering processes. ${ }^{6,14}$ It has been recently shown that neglecting this effect can lead to significant error in experimental signals. ${ }^{14}$

Figure 1(d) shows the temporal evolution of the (200) Bragg peak intensity by single-shot measurements under three different incident laser fluences $(F)$ as a function of the time delay between the probe pulse and the pump pulse. The intensity is normalized with respect to the value measured for each sample prior to laser excitation. At $27 \mathrm{~mJ} \mathrm{~cm}^{-2}, 40 \%$ of intensity is still detectable after $30 \mathrm{ps}$, while the intensity is almost reduced to zero within $\sim 15 \mathrm{ps}$ at $45 \mathrm{~mJ} \mathrm{~cm}^{-2}$, showing a strong dependence on the excitation fluence. The maximum contribution of the DWF to the reduction in the (200)-peak intensity is not likely to exceed $23 \%$ at the melting temperature $\left(T_{m}=1337 \mathrm{~K}\right)$, while it is more than $70 \%$ for the (420)-peak. ${ }^{13}$ We therefore pay most attention to the lower-order diffraction peaks as they capture the effects of loss of crystalline order during melting more directly.

The experimental data thus demonstrate that the film structure is evolving rapidly and is at least partially disordered after a few picoseconds, depending on the laser fluence. To determine the structural changes in the film reflected in the measured dynamics of Bragg peaks we apply 2T-MD. ${ }^{16}$ We used an MD cell containing $250 \mathrm{k}$ atoms with a size of $20.4 \times 20.4 \times 10.2 \mathrm{~nm}$ to represent a $10 \mathrm{~nm}\langle 100\rangle$ orientated $\mathrm{Au}$ film. The calculations provide the positions of all atoms in the film at each timestep of MD. We analyse the atomic structure as described below and link it to the UED data by calculating the real-space atomistic correlations for selected timesteps of MD to obtain the structure factor $\mathrm{S}(\mathrm{Q}){ }^{5}$ The time evolution of Bragg peaks can be obtained from $\mathrm{S}(\mathrm{Q})$ through a one-dimensional sine Fourier transform of the pair density function. ${ }^{8}$ As the quality criterion for the structure determination we use the agreement between the theoretical and experimental time evolutions of the Bragg peak intensities for several laser fluences. These strong nonlinear functions are sensitive to the parameters of the model and agreement between theory and experiment serves as a basis for faithful determination of the average parameters of the structure and the mechanism of solid/liquid phase transformation.

Running the 2T-MD requires solving the diffusion equation for the electron temperature simultaneously with the modified MD equations of motion, which incorporate an additional electron-ion energy exchange term via an inhomogeneous Langevin thermostat. ${ }^{9}$ To calculate the classical forces, an Au potential based on the embedded-atom method $(E A M)^{15}$ was used, which correctly reproduces the thermal and structural properties, such as the melting temperature and phonon spectra. The present formulation of the method takes into account only single elastic scattering events, which we confirmed experimentally. Both theoretical and experimental structure factors are integrated over the excited volume. We include longitudinal inhomogeneity, but we do not include inhomogeneity resulting from the transverse beam shape. Crucially, the time- and length-scales are the same for both experiment and theory. Therefore, we can compare the Bragg peak evolution directly and use detailed atomistic information from $2 \mathrm{~T}-\mathrm{MD}$ to reveal the mechanism of sample melting. 

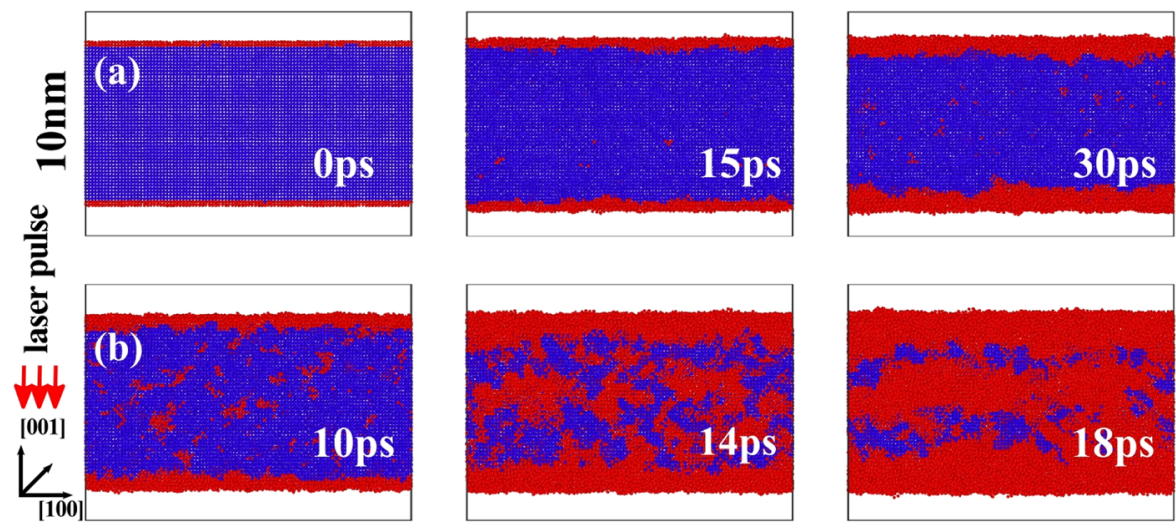

FIG. 3. Cross-sections of the atomistic simulations of ultrafast dynamics of single crystal Au films at selected simulation times for a $10 \mathrm{~nm}$ film (a) at $F_{\text {abs }}=3.0 \mathrm{~mJ} \mathrm{~cm}^{-2}$ (see supplementary material ${ }^{28}$ ) and (b) at $F_{\text {abs }}=4.5$ $\mathrm{mJ} \mathrm{cm}{ }^{-2}$ (see supplementary material $\left.^{28}\right)$. All atoms are colour-coded according to the measure of a degree of crystallinity given by the (nearest-neighbour averaged) centro-symmetry parameter $\Phi ;{ }^{10}$ blue atoms $(\Phi<0.45)$ have local crystalline structure, red atoms $(\Phi \geq 0.45)$ have highly disordered surroundings.
Below we discuss in more detail the main assumptions and parameters of our model. The full technical details of the calculations and the extension of the model to high fluence regime are published elsewhere. ${ }^{16} \mathrm{We}$ assume a well-defined electronic temperature $\left(T_{e}\right)$ at all times based on rapid thermalisation time of electrons ( $<100 \mathrm{fs}) .{ }^{17}$ The electronic temperature distribution after the laser pulse arrival is assumed to be spatially uniform throughout the simulation cell as we consider thin $\mathrm{Au}$ films (thinner than the electronic ballistic range or the optical penetration depth), ${ }^{18,19}$ but the consideration of a non-uniform distribution could be easily implemented. We also neglect the blast force ${ }^{20}$ resulting from the gradient of electronic temperature as we assume homogeneous excitations, but we could also include this effect in the model relatively easily.

The rate of energy transfer between the excited electrons and cold nuclei depends on the electronic specific heat $C_{e}$ and the effective electron-phonon (e-p) coupling $G$, both of which depend on the electronic temperature. ${ }^{21}$ The common use of the linear free electron gas specific heat leads to inaccurate $T_{e}$, as pointed out in Refs. 21 and 22. By computing it from the ab initio electronic internal energy: $C_{e}\left(T_{e}\right)=\left(\partial E_{e} / \partial T_{e}\right)$, we implicitly take into account both the effect of the electronic density of states (DOS) $)^{21}$ and its modification by elevated $T_{e}$. To evaluate $G\left(T_{e}\right)$, we used the experimental value of the e-p coupling constant at room temperature ${ }^{18}\left(G_{0}=2.1 \times 10^{16}\right.$ $\mathrm{W} \mathrm{m}{ }^{-3} \mathrm{~K}^{-1}$ ) and calculated the dependence on $T_{e}$ using $a b$ initio electronic DOS, as proposed in Refs. 21 and 23. It has recently been shown that this form of $G\left(T_{e}\right)$ is appropriate for metals. ${ }^{24}$ The values obtained for both $C_{e}\left(T_{e}\right)$ and $G\left(T_{e}\right)$ are similar to those by Lin et al. ${ }^{21}$ We neglected the effects of lattice temperature and structural changes on the electronphonon coupling as they are unknown for gold. ${ }^{25}$

The $a b$ initio calculations were performed using the ABINIT code, ${ }^{26,27}$ based on the local density approximation (LDA) and the norm-conserving pseudopotential method, where the $5 d$ and $6 s$ electrons are retained as valence electrons. The valence pseudo-wavefunctions are expanded in plane waves up to a cutoff of $60 \mathrm{Ha}$, and a $16 \times 16 \times 16$ Monkhorst-Pack $k$-points mesh is used.

The model described above allows one to calculate Bragg-peak intensities from atomic dynamics obtained by MD simulations for the given energy input, i.e., the energy absorbed by the Au film. The absorbed fluence $\left(F_{\text {abs }}\right)$ by the $\mathrm{Au}$ films can be estimated from the measured reflectivity $(\mathrm{R})$ and transmission (T) coefficients (our measurements give an absorption of 0.23 for our $10 \mathrm{~nm}$ films). In addition, there are energy losses due to dissipation into the supporting grid (via ballistic electrons ${ }^{19}$ ) and/or electron ejection, ${ }^{7}$ giving the following expression for the absorbed fluence:

$$
F_{\mathrm{abs}}=\eta_{\mathrm{loss}} \times(1-R(L)-T(L)) \times F,
$$

where $L$ is the thickness of the film. These losses strongly depend on a particular experimental setup. To assess these losses in our system, we performed independent timeresolved optical absorption measurements of $\mathrm{Au}$ thin films. The absorption spectra measured 30 ps after laser excitation, where hot electrons are well thermalized with the lattice, were compared with the spectra measured for the sample heated to temperatures from 293 to $450 \mathrm{~K}$. Comparison of the two measurements demonstrates that the lattice temperature increase after the laser excitation is much less than what would be expected if all absorbed fluence was used to heat up the sample, giving in our case $\eta_{\text {loss }}=0.5$. Therefore, we relate the incident fluences $F=1.0,27$, and $41 \mathrm{~mJ} \mathrm{~cm}^{-2}$, to the absorbed fluences $F_{\mathrm{abs}}=0.12,3.0$, and $4.5 \mathrm{~mJ} \mathrm{~cm}^{-2}$, respectively. In a case where $\eta_{\text {loss }}$ could not be precisely determined, $F_{\text {abs }}$ will become an adjustable parameter that would enter a fitting procedure.

Figure 2 demonstrates excellent agreement between the measured and calculated time evolutions of the Bragg peaks for the three analyzed fluences within the whole timedomain. Importantly, the model reproduces both the fast decay of Bragg intensity at short time-delays $(<5 \mathrm{ps})$ and the slower longer-term behaviour. The atomic structure is characterized by the degree of crystallinity given by the (nearestneighbour averaged) centro-symmetry parameter, ${ }^{10}$ local density, and local diffusivity.

At the lowest absorbed fluence of $0.12 \mathrm{~mJ} \mathrm{~cm}^{-2}$ (Fig. 2(a)), no structural changes were found in our 2T-MD simulations, and the Bragg peak intensity decrease is entirely due to the lattice temperature increase by $\sim 70 \mathrm{~K}$. The same temperature increase is observed in our optical absorption measurements for the same incident fluence, confirming our evaluation of $F_{\text {abs. }}$. For laser excitation at higher fluences, the reduction of the normalized intensities is more than $0.23 \%$, the maximum contribution of DWF to the reduction of the Bragg-peak intensity at $T_{m},{ }^{13}$ and the excitation-induced structural disorder is clearly seen in the snapshots of atomistic configurations in Fig. 3. We confirmed that the disordered structure corresponds to that of liquid gold for the same 
temperature and pressure by comparing radial distribution functions and self-diffusion coefficients both inside the disordered pockets and for the whole sample.

At the second fluence of $3.0 \mathrm{~mJ} \mathrm{~cm}^{-2}$ (Figs. 2(b) and $3(\mathrm{a})$ ), we observe pre-melting of the free surfaces and heterogeneous thermal melting by melt front propagation from the two free surfaces, but the film does not melt entirely and small regions of crystalline gold remain at $1.2 \mathrm{~ns}$, with a lattice temperature slightly above the melting point. At the highest fluence of $4.5 \mathrm{~mJ} \mathrm{~cm}^{-2}$ (Figs. 2(c) and 3(b)), we first observe a rapid expansion and surface pre-melting, accompanied by oscillations of the film thickness. This is followed by a rapid creation of homogeneously distributed small seeds of low-density molten phase (serving as nucleation sites) in an overheated state $\left(T_{m}<T_{i}<1.25 \times T_{m}\right)$, which are fleetingly created and destroyed, along with a melt front propagation $(6-10 \mathrm{ps})$ (see supplementary material $\left.{ }^{28}\right)$. These processes are responsible for the initial drop in the Bragg peak intensity. The further decrease in the Bragg peak intensity (10-20 ps), however, reflects the subsequent growth of the homogeneously distributed nucleation sites, creating low-density molten pockets, at ionic temperatures approaching the limit of crystal stability $\left(T_{i} \sim 1.25 \times T_{m}\right){ }^{8}$, until the sample melts entirely at around 20 ps. This complex interplay between homogeneous and heterogeneous melting processes explains the dynamics of the Bragg peaks and is consistent with recent theoretical predictions, 5,10 which confirm that our simulations give the correct melting processes and the correct percentage of melted regions.

One of the main goals of this paper is to extract the laser-induced disordering of the originally crystalline structure during the premelting or melting from the Bragg peak intensity changes. For this purpose, the low order Bragg peaks were targeted for analysis, since the higher order peaks are too sensitive to the DWF. We used 2T-MD to simulate the photo-excited thin gold films and computed the temporal evolution of Bragg peaks. This facilitated direct comparison with experiment which was carried out for three fluences. Although our results show a quantitative agreement between the measured and calculated time-evolution of Bragg peaks without any fitting procedure, the quality of this agreement depends on several factors. These include the absorbed fluence, the strength of the electron-phonon coupling, the electronic specific heat, and the choice of the interatomic potentials. In a case where $F_{\text {abs }}$ or the dependencies of $G$ are not known independently, they should enter as parameters in a standard fitting procedure similar to that used for static diffraction. Moreover, the dependence of the electron-phonon energy exchange term on the lattice temperature is currently discussed in the literature ${ }^{25}$ and could be easily incorporated in the model, allowing one to consider a variety of physical phenomena with very few fitting parameters. A reverse procedure, on the other hand, could provide these dependencies from accurate time-resolved diffraction data.

To summarise, the measured intensities in the UED experiments provide global information about the evolution of the crystalline order inside the sample, but do not deliver detailed atomistic information. The results of this work demonstrate that using 2T-MD allows us to quantitatively reproduce the time evolution of diffraction intensities and expose the atomistic dynamics of a photo-induced solid/liquid phase transition, which is equivalent to solving the inverse problem of structure determination in conventional diffraction measurements. Although the positions of atoms are not unique, the overall structural evolution is faithfully describing the atomic dynamics and the underlying mechanisms of melting behind the time evolution of Bragg peaks. This methodology can be applied to describe and understand photo-induced phase transitions in other metal nanofilms.

This work was supported by a specially promoted research (1900210) of Grant-in-Aid for Scientific Research from the MEXT, Japan. The authors are grateful to Y. Yoshida, H. Mori, and A. Takaoka for suggestions and discussions, and A. V. Rode and E. G. Gamaly for useful discussions. We acknowledge the UK's Materials Chemistry Consortium for providing HECToR computer resources (EPSRC grant: EP/F067496) and the UCL Legion High Performance Computing Facility, and associated support services. SD is funded by EPSRC under the M3S IDTC and CCFE.

${ }^{1}$ R. J. D. Miller, R. Ernstorfer, M. Harb, M. Gao, C. T. Hebeisen, H. JeanRuel, C. Lu, G. Moriena, and G. Sciaini, Acta Crystallogr. Sect. A 66, 137 (2010).

${ }^{2}$ G. Sciaini and R. J. D. Miller, Rep. Prog. Phys. 74, 096101 (2011).

${ }^{3}$ C.-Y. Ruan, Y. Murooka, R. K. Raman, R. A. Murdick, R. J. Worhatch, and A. Pell, Microsc. Microanal. 15, 323 (2009).

${ }^{4}$ B. J. Siwick, J. R. Dwyer, R. E. Jordan, and R. J. D. Miller, Science 302, 1382 (2003).

${ }^{5}$ Z. Lin and L. V. Zhigilei, Phys. Rev. B 73, 184113 (2006).

${ }^{6}$ R. Ernstorfer, M. Harb, C. T. Hebeisen, G. Sciaini, T. Dartigalongue, and R. J. D. Miller, Science 323, 1033 (2009).

${ }^{7}$ Y. Ping, A. A. Correa, T. Ogitsu, E. Draeger, E. Schwegler, T. Ao, K. Widmann, D. F. Price, E. Lee, P. T. Springer, D. Hanson, I. Koslow, D. Prendergast, G. Collins, and A. Ng, High Energy Density Phys. 6, 246 (2010).

${ }^{8}$ D. Ivanov and L. Zhigilei, Phys. Rev. B 68, 064114 (2003).

${ }^{9}$ D. Duffy and A. M. Rutherford, J. Phys.: Condens. Matter 19, 016207 (2007).

${ }^{10}$ Z. Lin, E. Leveugle, E. M. Bringa, and L. V. Zhigilei, J. Phys. Chem. C 114, 5686 (2010).

${ }^{11}$ Y. Gan and S. Jiang, J. Appl. Phys. 113, 073507 (2013).

${ }^{12}$ L. Reimer and H. Kohl, Transmission Electron Microscopy: Physics of Image Formation and Microanalysis, Springer Series in Optical Sciences (Springer, 2008).

${ }^{13}$ H. X. Gao and L.-M. Peng, Acta Crystallogr. Sect. A 55, 926 (1999).

${ }^{14}$ M. Ligges, I. Rajkovic, C. Streubuhr, T. Brazda, P. Zhou, O. Posth, C. Hassel, G. Dumpich, and D. von der Linde, J. Appl. Phys. 109, 063519 (2011).

${ }^{15}$ H. Sheng, M. Kramer, A. Cadien, T. Fujita, and M. Chen, Phys. Rev. B 83, 134118 (2011).

${ }^{16}$ S. L. Daraszewicz, Y. Giret, N. Naruse, Y. Murooka, J. Yang, D. M. Duffy, A. L. Shluger, and K. Tanimura, Phys. Rev. B 88, 184101 (2013).

${ }^{17}$ P. Musumeci, J. T. Moody, C. M. Scoby, M. S. Gutierrez, and M. Westfall, Appl. Phys. Lett. 97, 063502 (2010).

${ }^{18}$ J. Hohlfeld, S.-S. Wellershoff, J. Gudde, U. Conrad, V. Jahnke, and E. Matthias, Chem. Phys. 251, 237 (2000).

${ }^{19}$ T. Ogitsu, Y. Ping, A. Correa, B. Cho, P. Heimann, E. Schwegler, J. Cao, and G. W. Collins, High Energy Density Phys. 8, 303 (2012).

${ }^{20}$ J. Chen, W.-K. Chen, and P. M. Rentzepis, J. Appl. Phys. 109, 113522 (2011).

${ }^{21}$ Z. Lin, L. V. Zhigilei, and V. Cell, Phys. Rev. B 77, 075133 (2008).

${ }^{22}$ Y. Giret, A. Gellé, and B. Arnaud, Phys. Rev. Lett. 106, 155503 (2011).

${ }^{23}$ X. Y. Wang, D. M. Riffe, Y.-S. Lee, and M. C. Downer, Phys. Rev. B 50, 8016 (1994).

${ }^{24}$ J. B. Lee, K. Kang, and S. H. Lee, Mater. Trans. 52, 547 (2011).

${ }^{25}$ E. G. Gamaly and A. V. Rode, New J. Phys. 15, 013035 (2013). 
${ }^{26}$ X. Gonze, G.-M. Rignanese, M. Verstraete, J.-M. Beuken, Y. Pouillon, R. Caracas, F. Jollet, M. Torrent, G. Zerah, M. Mikami, Ph. Ghosez, M. Veithen, J.-Y. Raty, V. Olevano, F. Bruneval, L. Reining, R. Godby, G. Onida, D. R. Hamann, and D. C. Allan, Z. Kristallogr. 220, 558 (2005).

${ }^{27}$ X. Gonze, B. Amadon, P.-M. Anglade, J.-M. Beuken, F. Bottin, P. Boulanger, F. Bruneval, D. Caliste, R. Caracas, M. Côté, T. Deutsch, L. Genovese, Ph. Ghosez, M. Giantomassi, S. Goedecker, D. R. Hamann, P.
Hermet, F. Jollet, G. Jomard, S. Leroux, M. Mancini, S. Mazevet, M. J. T. Oliveira, G. Onida, Y. Pouillon, T. Rangel, G.-M. Rignanese, D. Sangalli, R. Shaltaf, M. Torrent, M. J. Verstraete, G. Zerah, and J. W. Zwanziger, Comput. Phys. Commun. 180, 2582 (2009).

${ }^{28}$ See supplementary material at http://dx.doi.org/10.1063/1.4847695 for visual animations of heterogeneous and homogeneous melting processes. 